\title{
Novel criticality in a model with absorbing states
}

\author{
Adam Lipowski* \\ Department of Physics, A. Mickiewicz University, Ul. Umultowska 85, 61-614 Poznań, Poland
}

(October 30, 2018)

\begin{abstract}
We study a one-dimensional model which undergoes a transition between an active and an absorbing phase. Monte Carlo simulations supported by some additional arguments prompted as to predict the exact location of the critical point and critical exponents in this model. The exponents $\delta=0.5$ and $z=2$ follows from random-walk-type arguments. The exponents $\beta=\nu_{\perp}$ are found to be non-universal and encoded in the singular part of reactivation probability, as recently discussed by H. Hinrichsen (cond-mat/0008179. A related model with quenched randomness is also studied.
\end{abstract}

\section{INTRODUCTION}

Recently, non-equilibrium phase transitions have been intensively studied in variety of models. Some attempts were also made to classify possible types of phase transitions into universality classes similarly to equilibrium transitions [1],2]. However, non-equilibrium transitions are much reacher and puzzling than equilibrium ones and their understanding is still far from complete.

A class of nonequlibrium models for which such a categorization seems most feasible are models with absorbing states [3]. For example, models with unique absorbing states most likely belong to the so-called directed-percolation (DP) universality class [4. Moreover, models with double (symmetric) absorbing states or with some conservation law in their dynamics belong to another universality class, the so-called parity-conserving (PC) universality class [5]. Although the above classification is best confirmed for one-dimensional models, it seems to apply to higher-dimensional models as well [6]. Moreover, models with more than two [7] or even infinitely many absorbing states also fall into the above universality classes. (Typically, models with infinitely many absorbing states fall into DP universality class, however, certain symmetries [8,9] or conservation laws [10] might change the criticality into PC.)

In the present paper we study certain one-dimensional models with infinitely many absorbing states. In the first part we study a model defined on a ladder-like lattice (ladder model) and our Monte Carlo simulations show that this model belongs to neither DP nor PC universality classes. Critical exponents $\delta$ and $z$ of this model appear to be rational numbers (contrary to the DP or PC criticality) and can be obtained using simple random-walk-type arguments. However, the exponents $\beta$ and $\nu_{\perp}(=\beta)$ appears to be non-universal. It turns out that their values are encoded in the singular part of the reactivation probability as was recently postulated in the context of related models by Hinrichsen [11].

Using certain property of our ladder model we can easily calculate the upper bound on the existence of the absorbing phase. Numerical simulations show that this bound most likely give exact location of the critical point in this model. In the final part of our paper we examine yet another but closely related model (single-chain model) for which we can obtain also the lower bound of the absorbing phase. It turns out that both bounds are the same which exactly locates the transition point in this model. The model studied in this part of our paper contains some quenched random variables and its critical behaviour is similar to that of the model studied in the main part of our paper.

\section{LADDER MODEL}

\section{A. Definition and basic properties}

Our model is a certain variant of models recently studied in some other contexts [9] 12 . It is defined on a onedimensional ladder-like lattice (see Fig. 17a). For each bond between the nearest-neighbouring sites we introduce a bond variable $w \in(-0.5,0.5)$. Introducing real parameters $r$ and $s>0$, we call a given site active when $w_{1} w_{2}\left|w_{3}\right|^{s}<r$, where $w_{1}$ and $w_{2}$ are intra-chain bond variables connected with this site and $w_{3}$ is the inter-chain bond variable (see Fig. 1 a). Otherwise, this site is called non-active. The model is driven by random sequential dynamics and when the active site is selected, we assign anew, with uniform probability, three neighbouring bond variables. Nonactive sites are not updated. 
First, let us notice that the model has a certain global up-down symmetry. Namely, reversing all bond variables $w \rightarrow-w$ we do not change the status of any site, i.e., active (non-active) sites remains active (non-active). The same symmetry appears in a closely related model which is defined on a single chain and where activity of a site is determined by the product of two intra-chain variables [9] (in the following we refer to this model as model A). It was suggested that this symmetry is responsible for the fact that the model A belongs to the PC universality class 9 . If so, the present model should also belong to this universality class. In the present paper we show, however, that the critical behaviour of this model is different. In our opinion, this result does not completely abolish the claims about the importance of the up-down symmetry. Namely, as we will show below, the change of the universality class in the present model comes from the very intricate mechanism: upon approaching the critical point the system might be regarded as composed of two weakly-interacting critical subsystems. We expect that in a generic case such a mechanism is absent and models of this kind which are endowed with the up-down symmetry should belong to the PC universality class.

This situation bears some similarity to the equilibrium statistical mechanics, where one expects that short-range interacting Ising models will generically belong to the Onsager universality class. It is known, however, that when certain additional interactions are included, the critical behaviour of an Ising model, which is then equivalent to the eight-vertex model, deviates from the generic case [13].

\section{B. Monte Carlo simulations}

To examine the properties of the above model we used standard Monte Carlo simulations. First, let us describe results for $s=2$. We calculated the steady-state density of active sites $\rho$. The fact that the model is defined on a one-dimensional lattice enabled us to examine systems of large size $L$ (up to $5 \cdot 10^{5}$ ). Also the simulation time $t_{\mathrm{MC}}$ was rather large and typically $t_{\mathrm{MC}} \sim 10^{5}-10^{6}$ (the unit of time is defined as a single, on average, update per lattice site). Our simulations show that for $r>0$ the density $\rho$ remains positive but as soon as $r$ becomes negative the system quickly reaches one of the absorbing states and thus $\rho=0$. A simple argument, similar to the one used for related models [9], shows that for $r<0$ the model gradually generate sites which remains permanently non-active. Indeed, let $r<0$ and a certain inter-chain bond $w_{3}$ satisfies the condition

$$
\left|w_{3}\right|<\sqrt{-r} /(0.5),
$$

then for each site connected to $w_{3}$ the absolute value of the product $w_{1} w_{2} w_{3}^{2}$ is smaller than $-r$, which implies $w_{1} w_{2} w_{3}^{2}>r$ and these sites remain permanently non-active. Since upon updating an active site there is a finite probability to satisfy (1), there is a finite rate of creation of such sites (a similar argument can be applied to an intra-chain bond). Thus, in agreement with numerical simulations, for $r<0$ the system is in the absorbing phase. Combined with the computationally observed property that for $r>0$ the density $\rho$ remains positive, it implies that $r=r_{\mathrm{c}}=0$ is the transition point for that model which separates active and absorbing phases. In addition, our simulations, which are described below, show that $r=0$ is actually a critical point of this model and is accompanied by typical power-law characteristics.

The first evidence of such a characteristic is shown in Fig. 2, which presents the logarithmic plot of $\rho$ as a function of $r$. The linearity of the plot for approximately three decades confirms the power-law behaviour $\rho \sim r^{\beta}$. At the same time, it confirms the location of the critical point $r_{\mathrm{c}}=0$. From the least-square analysis of these data we estimate $\beta=0.51(1)$.

Another quantity which we measured was the time evolution of the density $\rho$ for $r$ close to the critical point $r=r_{\mathrm{c}}$. One expects that at criticality $\rho \sim t^{-\delta}$ and deviations from the power-law behaviour appear off the critical point. The results are presented in Fig. 3. For $r=0$ the clear power-law behaviour is observed and from the slope of the data we estimate $\delta=0.50(1)$.

Other power-law characteristics are obtained from the finite-size analysis. In Fig. 1 we present the size dependence of the characteristic time $\tau$ defined as an average time needed for the system to reach an absorbing state (with a random initial configuration). Again, the best linearity is seen for $r=0$ and in this case we obtain $\tau \sim L^{z}$ with $z=2.01(1)$. Let us notice that this figure limits the allowed values of $r_{\mathrm{c}}$ to the very narrow range $-10^{-12}<r_{\mathrm{c}}<10^{-8}$.

Finally, Fig 5 presents the steady-state density $\rho$ as a function of size $L$. For $r=10^{-4}$ and $10^{-6}, \rho$ converges to the positive value but for $r=0$ it decays as $\rho \sim L^{-\beta / \nu_{\perp}}$ with the exponent $\beta / \nu_{\perp}=0.99(1)$.

For comparison with our model, we quote the values of these exponents for the one-dimensional DP [14] and PC [15] universality classes: $\beta=0.26486(\mathrm{DP}), 0.92(\mathrm{PC}) ; \delta=0.159464(\mathrm{DP}), 0.286(\mathrm{PC}) ; z=1.580745(\mathrm{DP}), 1.74(\mathrm{PC})$ and $\nu_{\perp}=1.096854(\mathrm{DP}), 1.83(\mathrm{PC})$. Thus, our results clearly places the model into a new universality class.

However, the obtained values of the exponents are not entirely unexpected. In the following we argue that at least the values of $\delta, z$ and of the ratio $\beta / \nu_{\perp}$ can be inferred from the properties of some other models. First, let us examine 
in more detail the model at the criticality, i.e., at $r=0$. Let us notice, that in this case it is only the sign of the expression $w_{1} w_{2} w_{3}^{2}$ which determines the state of a site. Since $w_{3}^{2}$ is always positive, it means that at the criticality this term is irrelevant and the state of a site is determined by the product $w_{1} w_{2}$, i.e., by the product of intra-chain variables. The same condition appears in model $\mathrm{A}$ and thus the present model at the criticality is equivalent to two non-interacting models A. Numerical evidence was already presented [9] that at $r=0$ model A is at the end-point of its critical phase which in A model appears in the range $0<r<r_{\mathrm{c}} \sim 0.027$. This critical phase is described by simple, random-walk related exponents: $\delta=0.5, z=2$ and $\beta / \nu_{\perp}=1$ [16]. On the basis of the above relation and in agreement with our simulations we obtain that these random-walk exponents are the critical exponents in our model. Let us emphasize that the critical phase in PC models exists in a certain range of a control parameter [17] and its (random-walk) criticality is described by a different set of exponents than the critical point of such models. In our model, there is only an isolated critical point which is described by the random-walk exponents.

The above relation with model $\mathrm{A}$ does not explain the value of the exponent $\beta$, which most likely equals 0.5 . This is because the relation with A model holds only at $r=0$ while the exponent $\beta$ describes the off-critical singularity. One can argue, however, that when $r$ is positive but small, then the interaction between these two models $\mathrm{A}$ is also small. Let us recall now, that for such $r$ model $\mathrm{A}$ is critical and thus the present model is equivalent to two weakly-interacting critical models A. It is interesting to observe that such an interaction is sufficient to destroy the criticality and to keep the system in the active phase with $\rho>0$. Qualitatively, we explain the large (comparing to model A) activity as follows: In models with absorbing states a non-active domain can be reactivated only through its boundaries. However, the already mentioned weak interaction can reactivate even the interior of such domains, which dramatically increases activity of the system.

\section{Reactivation probability}

In a recent paper Hinrichsen has shown that for certain models the singularity of the order parameter is determined by singularity of the reactivation probability $W$ [11]. His argument relies on the observation that $W$ usually scales linearly with the control parameter (i.e., a parameter which measures the distance from a critical point). Thus, in models where the density of active sites $\rho$ scales linearly with $W$, the singularity of $\rho$ as a function of the control parameter is solely a consequence of the singular behaviour of $W$. In this subsection we show that Hinrichsen's results extends also to the model examined in this paper.

Let us define the reactivation probability $W(r)$ as a probability that a given active site remains active after an update. To compute $W(r)$ we first calculate the probability density $P_{s}(z)$ that $w_{1} w_{2}\left|w_{3}\right|^{s}=z$ where $w_{1}, w_{2}$ and $w_{3}$ are independent and uniformly distributed on $(-0.5,0.5)$. Thus, we have to calculate

$$
P_{s}(z)=\int_{-1 / 2}^{1 / 2} d w_{1} \int_{-1 / 2}^{1 / 2} d w_{2} \int_{0}^{(1 / 2)^{s}} \frac{2}{s} \tilde{w}_{3}^{1 / s-1} \delta\left(z-w_{1} w_{2} \tilde{w}_{3}\right) d \tilde{w}_{3},
$$

where $\frac{2}{s} \tilde{w}_{3}^{1 / s-1}$ is the probability density of $\tilde{w}_{3}=\left|w_{3}\right|^{s}$, where $w_{3}$ is uniformly distributed on $(-1 / 2,1 / 2)$. The calculation of the integrals (2) is elementary. Perfoming integration over $w_{1}, w_{2}$ and then over $\tilde{w}_{3}$ we obtain

$P_{s}(z)=\frac{-4}{s} \int_{0}^{(1 / 2)^{s}} \tilde{w}_{3}^{1 / s-2} \ln \left(4|z| / \tilde{w}_{3}\right) \Theta\left(\tilde{w}_{3}-4|z|\right) d \tilde{w}_{3}=\frac{4}{s-1}\left\{2^{s-1}\left[\ln (4|z|)+s \ln (2)+\frac{s}{1-s}\right]+\frac{s}{s-1}(4|z|)^{1 / s-1}\right\}$

where $\Theta(x)$ is the unit step function. Having calculated $P_{s}(z)$, the reactivation probability $W(r)$ for positive $r$ is given as

$$
W(r)=\int_{(1 / 2)^{s+2}}^{r} P_{s}(z) d z=\frac{1}{2}+\frac{4}{s-1}\left\{2^{s-1}\left[r(\ln (4 r)-1)+\operatorname{sr}\left(\ln (2)+\frac{1}{1-s}\right)\right]+\frac{s^{2}}{4(s-1)}(4 r)^{1 / s}\right\} .
$$

From (4) one can see that $W(r)$ is indeed singular at the critical point $r=0$ and for $s>1 W(r) \sim r^{1 / s}$. One can see that the numerically found singularity of the order parameter with the exponent $\beta=1 / 2$ for $s=2$ corresponds to the same singularity of $W(r)$. Thus, the density $\rho$ in the vicinity of the critical point $r=0$ should scale linearly with the reactivation probability $W(r)$ and the singular behaviour is only due to the singular behaviour of $W(r)$ as a function of $r$.

To check the above arguments we performed simulations for $s=4$ and $1 / 2$. Let us notice that for $s=1 / 2$ the leading term is not $r^{1 / s}$ but $r \ln (4 r)$. In this case $\beta$ should be unity but the logarithmic corrections might substantially 
affect the scaling behaviour. The results are presented in Fig. 2. As estimated from the slope of our data, $\beta=0.26(1)$ for $s=4$ and $\beta=1.3(2)$ for $s=1 / 2$. For $s=4$ the exponent $\beta$ is very close to $1 / 4$ which clearly confirms our arguments. For $s=0.5$ our data are less accurate mainly due to very large relaxation time. Moreover, the logarithmic corrections might be responsible for the fact that the 'true' scaling is not clearly seen.

Let us also notice that at the critical point $r=0$ only the sign matters and the behaviour of the ladder model is independent on $s$. Thus, the remaining exponents $\delta, z$ and the ratio $\beta / \nu_{\perp}$ must be the same as in the $s=2$ case.

\section{SINGLE-CHAIN MODEL WITH QUENCHED DISORDER}

In this section we examine a simple model which might help us to understand the behaviour of the ladder model studied in the previous section. The model is defined on a single chain (see Fig. 1 b). In addition to bond variables $-1 / 2<w<1 / 2$ there are on-site quenched variables $0<v<1$. A given site is defined as active if $w_{1} w_{2} v<r$, where $w_{1}$ and $w_{2}$ are variables on bonds connected to this site and $v$ is the corresponding site variable. Once initially selected, $v$-variables remain unchanged during the evolution of this model (evolving variables are only bond variables). (The already discussed model A corresponds to the case $v=1$ on each site.)

It is elementary to prove the following properties of the this model:

(i) Similarly to the model studied in the previous section the model for $r<0$ generates with a finite rate sites which remain permanently non-active. It means that for $r<0$ the model is in the absorbing phase.

(ii) Let $r>0$. Let us consider a site for which $v<4 r$. It is obvious that for any choice of bond variables attached to it this site remains permanently active. Since in the thermodynamic limit a finite fraction $4 r$ of sites has $v<4 r$, we obtain that for $r>0$ the model is in the active phase.

As a conclusion, we obtain that $r=0$ separates active and absorbing phases of this model. Let us also notice that for $r=0$ the model is equivalent to the model A (i.e., $v$ variables are irrelevant), which was shown to be critical at that point [9]. Moreover, the fraction $4 r$ of permanently active sites decreases linearly to zero which suggests that in this model $\beta=1[18]$.

Monte Carlo simulations of this model strongly suggest that indeed $\beta=1$ (see Fig. 2). Similarly to the ladder model, at $r=0$ only the sign matters and $v$-variables are irrelevant. Thus, at criticality the behaviour of this model is the same as of the A model at $r=0$. Numerical simulations of the latter model show [9] that in this case $z=2$ and $\delta=1 / 2$ as in the ladder model.

\section{CONCLUSIONS}

In conclusion, we examined a class of models with infinitely many absorbing states. Critical exponents $\delta$, $z$ and $\beta / \nu_{\perp}$ take random-walk values. However, the exponents $\beta$ and $\nu_{\perp}$ are non-universal. This non-universality is related to the singular behaviour of the reactivation probability $W(r)$. Thus, when as a control parameter of the model we choose $W(r)$ rather than $r$, the order parameter would have the universal exponent $\beta=1$. It would be interesting to examine this model using, for example, field-theory methods [19] and to check whether some other universality classes among models with absorbing states are possible.

\section{ACKNOWLEDGMENTS}

I thank Haye Hinrichsen for very stimulating correspondence and the Department of Mathematics of the Heriot-Watt University (Edinburgh, Scotland) for allocation of computer time.

* e-mail: lipowski@main.amu.edu.pl

[1] For a recent review with a large number of references see: H. Hinrichsen, Critical Phenomena in Nonequilibrium Systems, preprint: cond-mat/0001070.

[2] Evans, Brazil. J. Phys. 32, 42 (2000).

[3] J. Marro and R. Dickman, Nonequilibrium Phase Transitions in Lattice Models, (Cambridge University Press, Cambridge, 1999). 
[4] P. Grassberger, Z. Phys. B 47, 365 (1982). H. K. Janssen, Z. Phys. B 42, 151 (1981).

[5] P. Grassberger, F. Krause and T. von der Twer, J. Phys. A 17, L105 (1984). I. Jensen, Phys. Rev. E 50, 3623 (1994). H. Hinrichsen, Phys. Rev. E 55, 219 (1997). H. Takayasu and A. Yu. Tretyakov, Phys. Rev. Lett. 68, 3060 (1992). A. Lipowski, J. Phys. A 29, L355 (1996).

[6] In higher dimensions PC models are divided into two distinct universality classes (see, e.g., 洎] for details).

[7] H. K. Janssen, Phys. Rev. Lett. 78, 2890 (1997).

[8] W. Hwang and H. Park, Phys. Rev. E 59, 4683 (1999).

[9] A. Lipowski, Phys. Rev. E 62, 3356 (2000).

[10] M. C. Marques and J. F. F. Mendes, Eur. Phys. J. B 12, 123 (1999).

[11] H. Hinrichsen, e:preprint (cond-mat/0008179).

[12] A. Lipowski and M. Lopata, Phys. Rev. E 60, 1516 (1999). A. Lipowski, Phys. Rev. E 60, R6255 (1999).

[13] R. J. Baxter, Exactly Solved Models in Statistical Mechanics, (Academic Press, New York, 1982).

[14] I. Jensen, J. Phys. A 32, 5233 (1999).

[15] see, e.g., I. Jensen, Phys. Rev. E 50, 3623 (1994).

[16] P. A. Alemany and D. ben-Avraham, Phys. Lett A 206, 18 (1995). See also: E. Carlon, M. Henkel and U. Schollwoeck, preprint (cond-mat/9912347).

[17] Usually the entire non-active phase of PC models is critical. The only example, of which we are aware of, where this phase terminates at a certain value of a control parameter is model $\mathrm{A}$.

[18] One might expect that for small but positive $r$ the activity is localized only around these permanently active sites.

[19] J. Cardy and U. C. Täuber, Phys. Rev. Lett 77, 4780 (1996). D. C. Mattis and M. L. Glasser, Rev. Mod. Phys. 70, 979 (1998).

FIG. 1. (a) The ladder model. The site $(\bullet)$ is active when $w_{1} w_{2}\left|w_{3}\right|^{s}<r$. An update of an active site replaces all three neighbouring bonds (o) with uniformly distributed random numbers from the interval $(-0.5,0.5)$. (b) The single-chain model. The site is active when $w_{1} w_{2} v<r$ and its update replaces only $w_{1}$ and $w_{2}$.

FIG. 2. The log-log plot of the density of active sites $\rho$ as a function of $r$. The linear size $L$ in this simulations varied from $L=5 \cdot 10^{4}$ to $2 \cdot 10^{6}$. The solid lines (least-square fit) have slopes corresponding to $\beta=0.26(1)$ (for $\left.s=4\right), 0.51(1)($ for $s=2$ ), $1.3(2)$ (for $s=0.5$ ) and $0.99(2)$ for the single-chain model.

FIG. 3. The time evolution of the density $\rho$ for (from top to bottom) $r=10^{-4}, 10^{-6}, 0,-10^{-10},-10^{-8},-10^{-6}$, and $-10^{-4}$. The simulations were made for $s=2$ and $L=10^{5}$ and we checked that for the examined time scale our data are basically size-independent. These data limit $r_{\mathrm{c}}$ to the range $-10^{-10}<r_{\mathrm{c}}<10^{-6}$. Even tighter bounds follow from Fig. 1 .

FIG. 4. The size dependence of the characteristic time $\tau$ for (from top to bottom): $r=10^{-6}, 10^{-8}, 0,-10^{-12},-10^{-8}$, $-10^{-6}$, and $-10^{-4}$. The straight line has a slope corresponding to $z=2.01$. Each point is an average of 100 independent runs and $s=2$.

FIG. 5. The size dependence of the steady-state density $\rho$ for $r=10^{-4}(\square), 10^{-6}(+)$ and $0(\diamond)$. The straight line has a slope corresponding to $\beta / \nu_{\perp}=0.99(2)(s=2)$. 


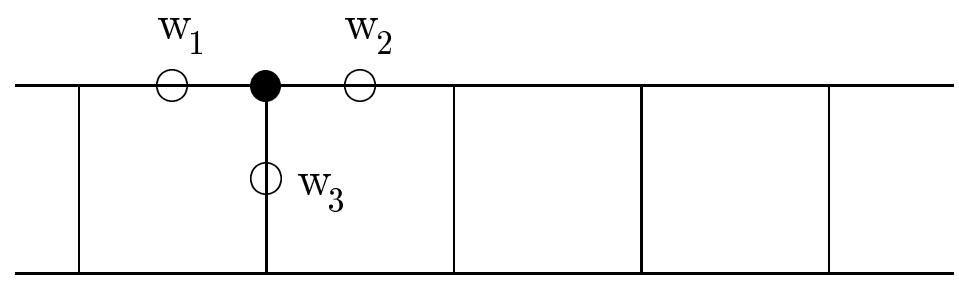

(a)

$\stackrel{\mathrm{W}_{1}}{{ }^{\prime}} \stackrel{\mathrm{V}}{\bullet} \stackrel{\mathrm{W}}{2}^{\mathrm{W}}$

(b)

Fig.1 


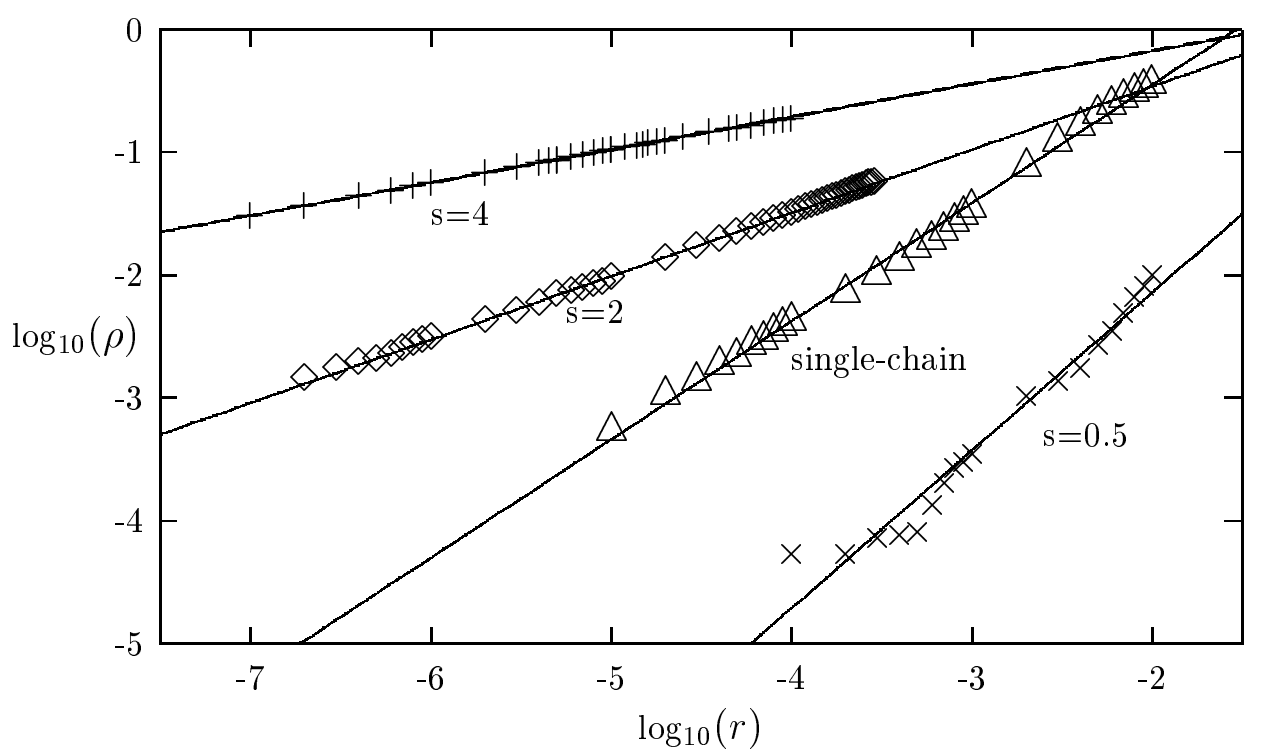

Fig.2 


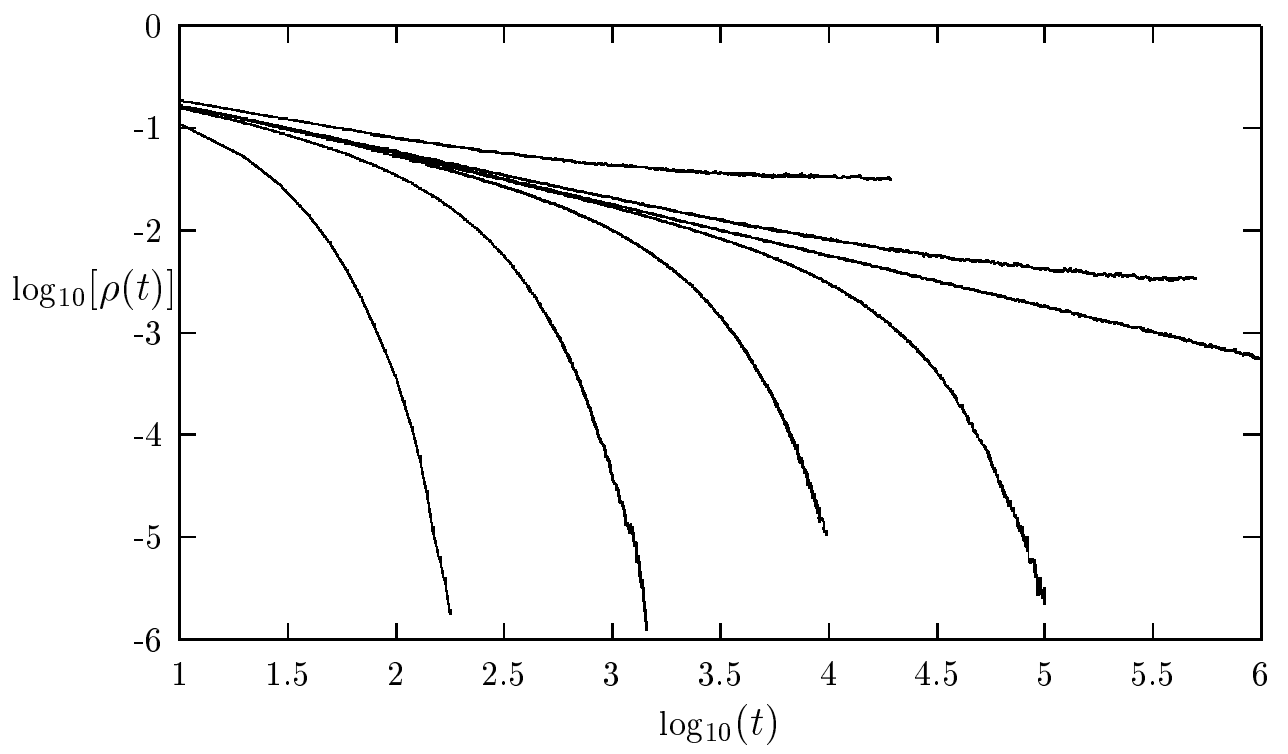

Fig.3 


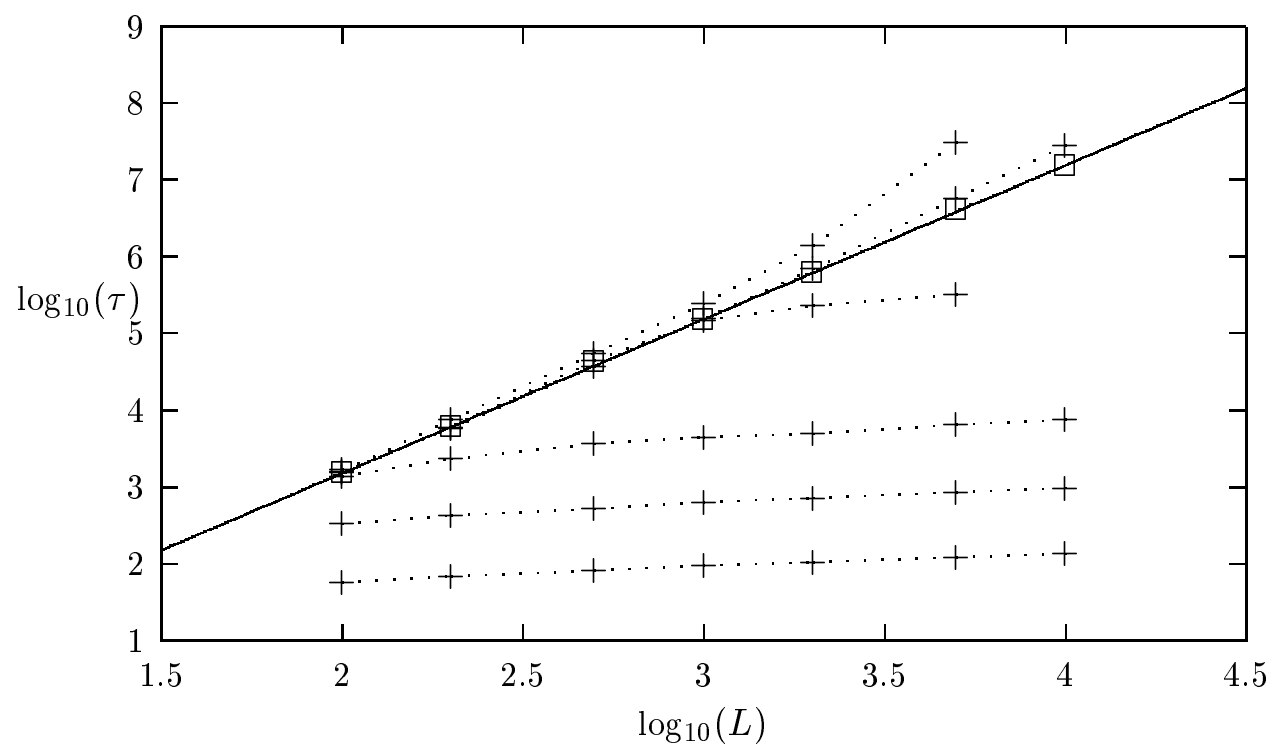

Fig.4 


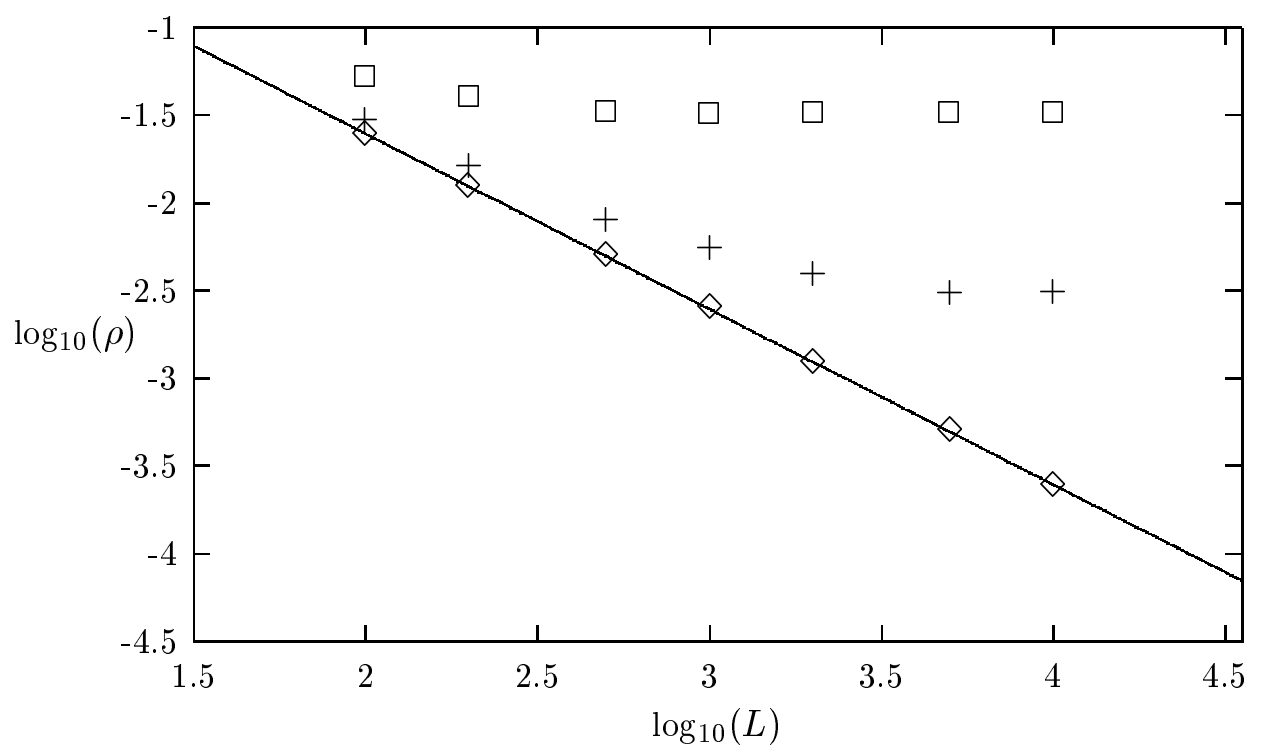

Fig.5 